\title{
Bethe-Salpeter-Motivated Modelling of Pseudo-Goldstone Pseudoscalar Mesons
}

\section{Wolfgang Lucha*}

Institute for High Energy Physics, Austrian Academy of Sciences, Nikolsdorfergasse 18, A-1050 Vienna, Austria

E-mail: Wolfgang. Lucha@oeaw.ac.at

\begin{abstract}
We apply a description of bound states of fermion and antifermion by means of our approximation to the Bethe-Salpeter formalism that retains part of the information on relativistic effects provided by the full fermion propagator to the lightest pseudoscalar mesons. Therein, the pseudo-Goldstone nature of the latter quark-antiquark bound states is taken into account by appropriately formulated effective interactions. Scrutinizing the predictions of this bound-state approach for meson masses, decay constants and in-meson condensates by relying on a generalized Gell-Mann-Oakes-Renner relation shows that the light-quark-mass values required for agreement are all in the right ballpark.
\end{abstract}

XIII Quark Confinement and the Hadron Spectrum - Confinement2018

31 July - 6 August 2018

Maynooth University, Ireland

${ }^{*}$ Speaker. 


\section{Interpretation of the Lightest Pseudoscalar Mesons as (Pseudo) Goldstone Bosons}

Spontaneous breakdown of the chiral symmetries of quantum chromodynamics (QCD) implies the presence of massless bosons, identified with the ground-state pseudoscalar mesons, with masses due to these symmetries' further, explicit breaking. As a proof of concept, we study the treatment of such pseudo-Goldstone bosons by a simple approximation [1-5] to the Bethe-Salpeter equation [6].

\section{Bethe-Salpeter Equation for Fermion-Antifermion States in Instantaneous Limit}

Consider some boson bound state $|B(P)\rangle$ of mass $\widehat{M}_{B}$ and momentum $P$, composed of a fermion and an antifermion with individual coordinates $x_{1,2}$, individual momenta $p_{1,2}$, center-of-momentum coordinate $X$, relative coordinate $x$, total momentum $P$, and relative momentum $p$, clearly related by

$$
x \equiv x_{1}-x_{2}, \quad P \equiv p_{1}+p_{2}, \quad P^{2}=\widehat{M}_{B}^{2} .
$$

The Bethe-Salpeter formalism describes the bound state $|B(P)\rangle$ by its Bethe-Salpeter amplitude, in momentum space defined, in terms of the Dirac field operators $\psi_{1,2}\left(x_{1,2}\right)$ of the two constituents, by

$$
\Phi(p, P) \equiv \exp (\mathrm{i} P X) \int \mathrm{d}^{4} x \exp (\mathrm{i} p x)\left\langle 0\left|\mathrm{~T}\left(\psi_{1}\left(x_{1}\right) \bar{\psi}_{2}\left(x_{2}\right)\right)\right| B(P)\right\rangle .
$$

This Bethe-Salpeter amplitude satisfies the homogeneous Bethe-Salpeter equation [6] that, in turn, involves both the appropriate interaction kernel and the propagators of the bound-state constituents. In Lorentz-covariant settings, the full propagator $S_{i}(p)$ of any spin- $\frac{1}{2}$ fermion $i$ can be represented in terms of two Lorentz-scalar functions, e.g., mass $M_{i}\left(p^{2}\right)$ and wave-function renormalization $Z_{i}\left(p^{2}\right)$, obtained as solutions to the Dyson-Schwinger equation for the fermion's two-point Green function:

$$
S_{i}(p)=\frac{\mathrm{i} Z_{i}\left(p^{2}\right)}{\not p-M_{i}\left(p^{2}\right)+\mathrm{i} \varepsilon}, \quad \not p \equiv p^{\mu} \gamma_{\mu}, \quad \varepsilon \downarrow 0, \quad i=1,2 .
$$

Some time ago, we devised a (Salpeter-equation-generalizing) three-dimensional reduction [1] of the Poincaré-covariant Bethe-Salpeter equation, enabled by keeping in fermion propagators only terms linear in $p_{0}$. The latter, together with the assumption of instantaneity of all interactions among the bound-state constituents, suffices to formulate bound-state equations for Salpeter amplitudes [7]

$$
\phi(\boldsymbol{p}) \propto \int \mathrm{d} p_{0} \Phi(p, P) .
$$

In terms of its bound-state constituents' free energies and projectors onto positive/negative energies,

$$
E_{i}(\boldsymbol{p}) \equiv \sqrt{\boldsymbol{p}^{2}+M_{i}^{2}\left(\boldsymbol{p}^{2}\right)}, \quad \Lambda_{i}^{ \pm}(\boldsymbol{p}) \equiv \frac{E_{i}(\boldsymbol{p}) \pm \gamma_{0}\left[\boldsymbol{\gamma} \cdot \boldsymbol{p}+M_{i}\left(\boldsymbol{p}^{2}\right)\right]}{2 E_{i}(\boldsymbol{p})},
$$

and induced interaction kernel $K(\boldsymbol{p}, \boldsymbol{q})$, our center-of-momentum-frame bound-state equation reads

$$
\begin{aligned}
\phi(\boldsymbol{p})=Z_{1}\left(\boldsymbol{p}^{2}\right) Z_{2}\left(\boldsymbol{p}^{2}\right) \int \frac{\mathrm{d}^{3} q}{(2 \pi)^{3}} & \left(\frac{\Lambda_{1}^{+}(\boldsymbol{p}) \gamma_{0}[K(\boldsymbol{p}, \boldsymbol{q}) \phi(\boldsymbol{q})] \Lambda_{2}^{-}(\boldsymbol{p}) \gamma_{0}}{\widehat{M}_{B}-E_{1}(\boldsymbol{p})-E_{2}(\boldsymbol{p})}\right. \\
- & \left.\frac{\Lambda_{1}^{-}(\boldsymbol{p}) \gamma_{0}[K(\boldsymbol{p}, \boldsymbol{q}) \phi(\boldsymbol{q})] \Lambda_{2}^{+}(\boldsymbol{p}) \gamma_{0}}{\widehat{M}_{B}+E_{1}(\boldsymbol{p})+E_{2}(\boldsymbol{p})}\right) .
\end{aligned}
$$

The normalization of the Salpeter amplitude $\phi(\boldsymbol{p})$ will, of course, reflect that of the state $|B(P)\rangle$ entering its definition. For the latter normalization, we adhere to the relativistically covariant choice

$$
\left\langle B(P) \mid B\left(P^{\prime}\right)\right\rangle=(2 \pi)^{3} 2 P_{0} \boldsymbol{\delta}^{(3)}\left(\boldsymbol{P}-\boldsymbol{P}^{\prime}\right) .
$$


Neglecting, for one reason or the other, the impact of the interaction kernel, this yields the condition (involving a trace over our bound-state constituents' spinor, flavour, and colour degrees of freedom)

$$
\int \mathrm{d}^{3} p \operatorname{Tr}\left[\phi^{\dagger}(\boldsymbol{p}) \frac{\gamma_{0}\left[\boldsymbol{\gamma} \cdot \boldsymbol{p}+M_{1}\left(\boldsymbol{p}^{2}\right)\right]}{E_{1}(\boldsymbol{p})} \phi(\boldsymbol{p})\right]=(2 \pi)^{3} 2 P_{0} .
$$

\section{Application Suggesting Itself: Two Bound-State Constituents of Identical Flavour}

Now, let us adapt our general instantaneous Bethe-Salpeter formalism [1] to just those physical systems we are actually interested in: bound states of a quark and an antiquark of precisely the same mass - tantamount, as far as only the strong interactions are taken into account, to bound states of a quark and its own antiquark. For this special case, we may drop the flavour-related subscript $i=1,2$ in our framework, whence the instantaneous Bethe-Salpeter equation (2.2) simplifies (a little bit) to

$$
\begin{aligned}
\phi(\boldsymbol{p})=Z^{2}\left(\boldsymbol{p}^{2}\right) \int \frac{\mathrm{d}^{3} q}{(2 \pi)^{3}} & \left(\frac{\Lambda^{+}(\boldsymbol{p}) \gamma_{0}[K(\boldsymbol{p}, \boldsymbol{q}) \phi(\boldsymbol{q})] \Lambda^{-}(\boldsymbol{p}) \gamma_{0}}{\widehat{M}_{B}-2 E(\boldsymbol{p})}\right. \\
- & \left.\frac{\Lambda^{-}(\boldsymbol{p}) \gamma_{0}[K(\boldsymbol{p}, \boldsymbol{q}) \phi(\boldsymbol{q})] \Lambda^{+}(\boldsymbol{p}) \gamma_{0}}{\widehat{M}_{B}+2 E(\boldsymbol{p})}\right) .
\end{aligned}
$$

Clearly, the spin-parity-charge-conjugation assignment of any pseudoscalar bound state formed by spin- $\frac{1}{2}$ fermion and spin- $\frac{1}{2}$ antifermion is given by $J^{P C}=0^{-+}$. The most general Salpeter amplitude $\phi(\boldsymbol{p})$ of any such state may be expanded into only two independent Lorentz-scalar components, say, $\varphi_{1,2}(\boldsymbol{p})$. Recalling its colour factor, for a bound state of quark and its antiquark this expansion reads

$$
\begin{aligned}
& \phi(\boldsymbol{p})=\frac{1}{\sqrt{3}}\left[\varphi_{1}(\boldsymbol{p}) \frac{\gamma_{0}\left[\boldsymbol{\gamma} \cdot \boldsymbol{p}+M\left(\boldsymbol{p}^{2}\right)\right]}{E(\boldsymbol{p})}+\varphi_{2}(\boldsymbol{p})\right] \gamma_{5}, \\
& 4 \int \mathrm{d}^{3} p\left[\varphi_{1}^{*}(\boldsymbol{p}) \varphi_{2}(\boldsymbol{p})+\varphi_{2}^{*}(\boldsymbol{p}) \varphi_{1}(\boldsymbol{p})\right]=(2 \pi)^{3} 2 P_{0} .
\end{aligned}
$$

At that stage, the only element still lacking is the Bethe-Salpeter kernel $K(\boldsymbol{p}, \boldsymbol{q})$, with regard to its Dirac structure and its dependence on the momenta $\boldsymbol{p}$ and $\boldsymbol{q}$. We tackle this problem in two steps.

\subsection{Dirac Structure of the Bethe-Salpeter Interaction Kernel by Sticking to Fierz Invariance}

We base the determination of the kernel $K(\boldsymbol{p}, \boldsymbol{q})$ on our trust in Fierz symmetries and rely for its Dirac structure on a linear combination corresponding to an eigenstate under Fierz transformations:

$$
K(\boldsymbol{p}, \boldsymbol{q}) \phi(\boldsymbol{q}) \propto V(\boldsymbol{p}, \boldsymbol{q})\left[\gamma_{\mu} \phi(\boldsymbol{q}) \gamma^{\mu}+\gamma_{5} \phi(\boldsymbol{q}) \gamma_{5}-\phi(\boldsymbol{q})\right] .
$$

Accordingly, all underlying effective interactions are subsumed by a single Lorentz-scalar potential function, $V(\boldsymbol{p}, \boldsymbol{q})$. Assuming the latter to be of convolution type and to be compatible with spherical symmetry, that is, $V(\boldsymbol{p}, \boldsymbol{q})=V\left((\boldsymbol{p}-\boldsymbol{q})^{2}\right)$, allows us to split off all reference to angular variables and to reduce our bound-state equation (3.1) to a system of equations for the radial factors $\varphi_{1,2}(p)$ of the independent components $\varphi_{1,2}(\boldsymbol{p})$, depending on the moduli $p \equiv|\boldsymbol{p}|, q \equiv|\boldsymbol{q}|$ of the momenta $\boldsymbol{p}$ and $\boldsymbol{q}$, with all interactions encoded by a yet to be found configuration-space central potential $V(r), r \equiv|x|$ :

$$
\begin{aligned}
& E(p) \varphi_{2}(p)+\frac{2 Z^{2}\left(p^{2}\right)}{\pi p} \int_{0}^{\infty} \mathrm{d} q q \mathrm{~d} r \sin (p r) \sin (q r) V(r) \varphi_{2}(q)=\frac{\widehat{M}_{B}}{2} \varphi_{1}(p), \\
& E(p) \varphi_{1}(p)=\frac{\widehat{M}_{B}}{2} \varphi_{2}(p) .
\end{aligned}
$$




\subsection{Momentum Dependence of our Bethe-Salpeter Interaction Kernel by Utilizing Inversion}

The two (in general, coupled) Eqs. (3.3) constitute an eigenvalue problem, with the bound-state masses $\widehat{M}_{B}$ as eigenvalues, for bound states specified, in momentum-space representation, by the set of radial wave functions $\varphi_{1,2}(p)$. For vanishing eigenvalue, that is, for $\widehat{M}_{B}=0$, Eqs. (3.3) decouple: Eq. (3.3b) forces $\varphi_{1}(p)$ to vanish, i.e., $\varphi_{1}(\boldsymbol{p})=0$. Thus, the corresponding Salpeter amplitude reads

$$
\phi(p)=\frac{1}{\sqrt{3}} \varphi_{2}(p) \gamma_{5} .
$$

In configuration-space representation, denoting the free term by $T(r)$, Eq. (3.3a) then simplifies to a relation enabling us $[8,9]$ to find the potential in action, $V(r)$, provided we know one solution $\varphi_{2}(r)$ :

$$
T(r)+V(r) \varphi_{2}(r)=0 \quad \Longrightarrow \quad V(r)=-\frac{T(r)}{\varphi_{2}(r)} .
$$

In order to get hold of, at least, one of the desired solutions, we exploit the relationship between the full quark propagator $S(p)$ - obtainable as solution to the quark Dyson-Schwinger equation and the Bethe-Salpeter amplitude $\Phi(p, 0)$ of (flavour-nonsinglet) pseudoscalar mesons arising from the (renormalized) axial-vector Ward-Takahashi identity of QCD in the chiral limit [10]: the sought relationship (in its Euclidean-space formulation indicated by underlined quantities) reads [2,11-14]

$$
\Phi(\underline{k}, 0) \propto \frac{Z\left(\underline{k}^{2}\right) M\left(\underline{k}^{2}\right)}{\underline{k}^{2}+M^{2}\left(\underline{k}^{2}\right)} \underline{\gamma}_{5}+\text { subleading contributions . }
$$

Just for the sake of illustration, let us follow the path sketched above by starting from a solution for the chiral-quark propagator found in Ref. [15] on the basis of a particular QCD-motivated ansatz for the effective interactions entering in the quark Dyson-Schwinger equation: the conversion of the propagator functions $M(\underline{k})$ and $Z(\underline{k})$ redrawn in Fig. 1, by means of Eq. (3.6), to the massless-meson Salpeter amplitude of Fig. 2 entails, via the inversion (3.5), the interquark potential plotted in Fig. 3.

\section{Basic Pseudoscalar-Meson Features in a Gell-Mann-Oakes-Renner-type Relation}

With the explicit behaviour of the effective interquark potential $V(r)$ at our disposal, we are in a position to embark on the intended simplified description of meson properties: for $\widehat{M}_{B} \neq 0$, inserting any of Eqs. (3.3) into the other, takes us to a single eigenvalue equation for eigenvalues $\widehat{M}_{B}^{2}$ [2-5,17], which can be easily solved by expanding its solutions over suitable bases in function space [18-25].

Matching residues of pseudoscalar-meson poles in the axial-vector Ward-Takahashi identity of QCD gives a Gell-Mann-Oakes-Renner-resembling [26] relation [10] linking, besides meson mass $\widehat{M}_{B}$ and two quark masses, both decay constant $f_{B}$ and in-hadron condensate $\mathbb{C}_{B}$ of the pseudoscalar bound state $|B(P)\rangle$, defined, in terms of quark fields $\psi_{f}(x)$ (exhibiting the flavour index $f=1,2$ ), by

$$
\begin{array}{r}
\left\langle 0\left|: \bar{\psi}_{1}(0) \gamma_{\mu} \gamma_{5} \psi_{2}(0):\right| B(P)\right\rangle=\mathrm{i} f_{B} P_{\mu} \quad \Longrightarrow \quad f_{B} \propto \int \mathrm{d}^{3} p \operatorname{Tr}\left[\gamma_{0} \gamma_{5} \phi(\boldsymbol{p})\right], \\
\left\langle 0\left|: \bar{\psi}_{1}(0) \gamma_{5} \psi_{2}(0):\right| B(P)\right\rangle \equiv \mathbb{C}_{B} \propto \int \mathrm{d}^{3} p \operatorname{Tr}\left[\gamma_{5} \phi(\boldsymbol{p})\right] .
\end{array}
$$

Sticking still to the idealized case of bound-state constituents of equal mass $m$, this relation becomes

$$
f_{B} \widehat{M}_{B}^{2}=2 m \mathbb{C}_{B}
$$




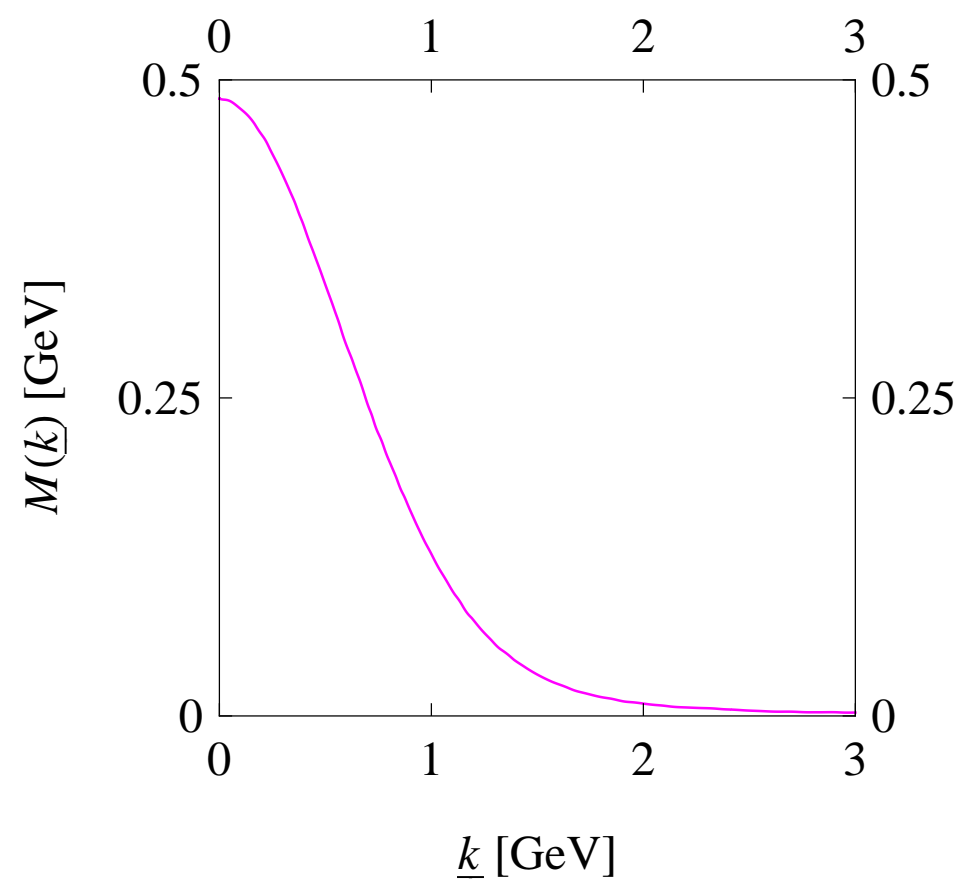

(a)

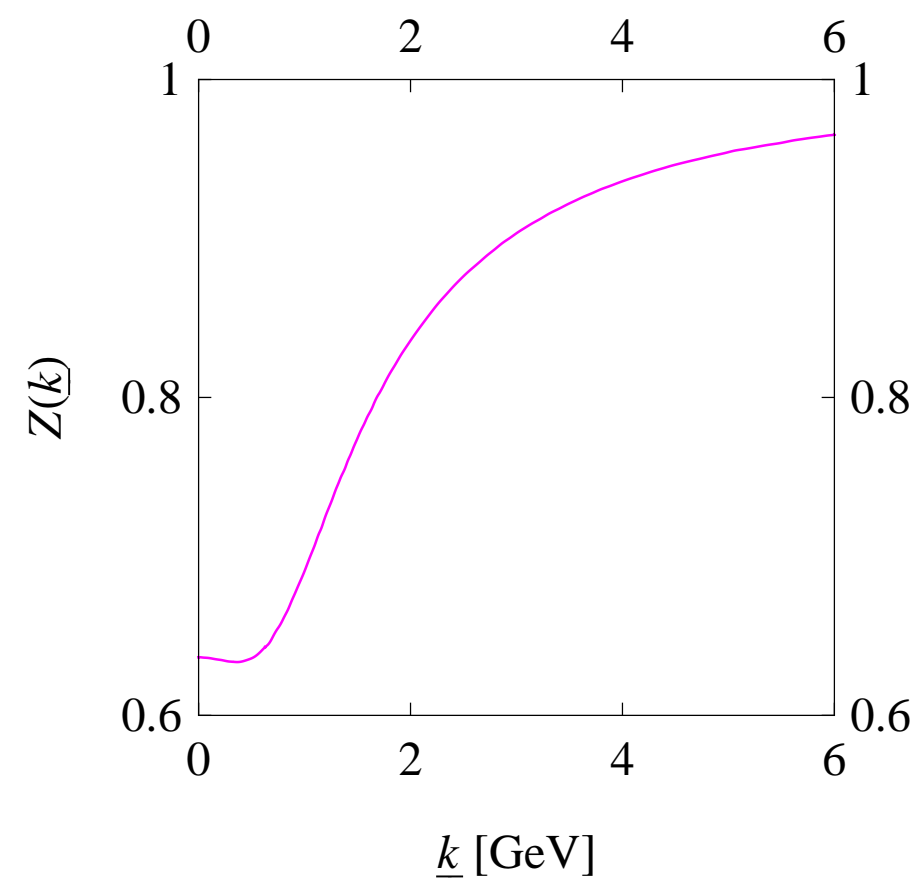

(b)

Figure 1: Dependence, on the Euclidean momentum $\underline{k}$, of both (a) mass function $M(\underline{k})$ and (b) wave-function renormalization function $Z(\underline{k})$ entering in the quark propagator (2.1) in the chiral limit, extracted and redrawn [2,4] from Fig. 1 of Ref. [16], emerging as solution to the Dyson-Schwinger equation for the quark two-point Green function imitating the impact of truncated Dyson-Schwinger equations by QCD-inspired ansätze [15]. 


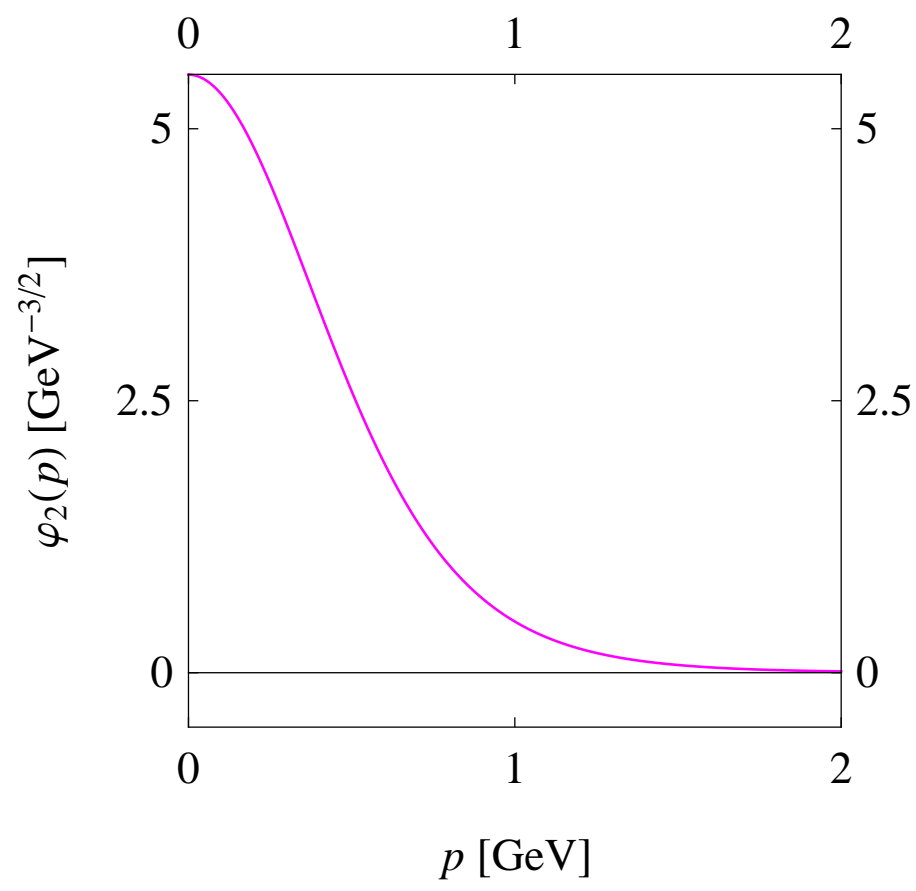

(a)

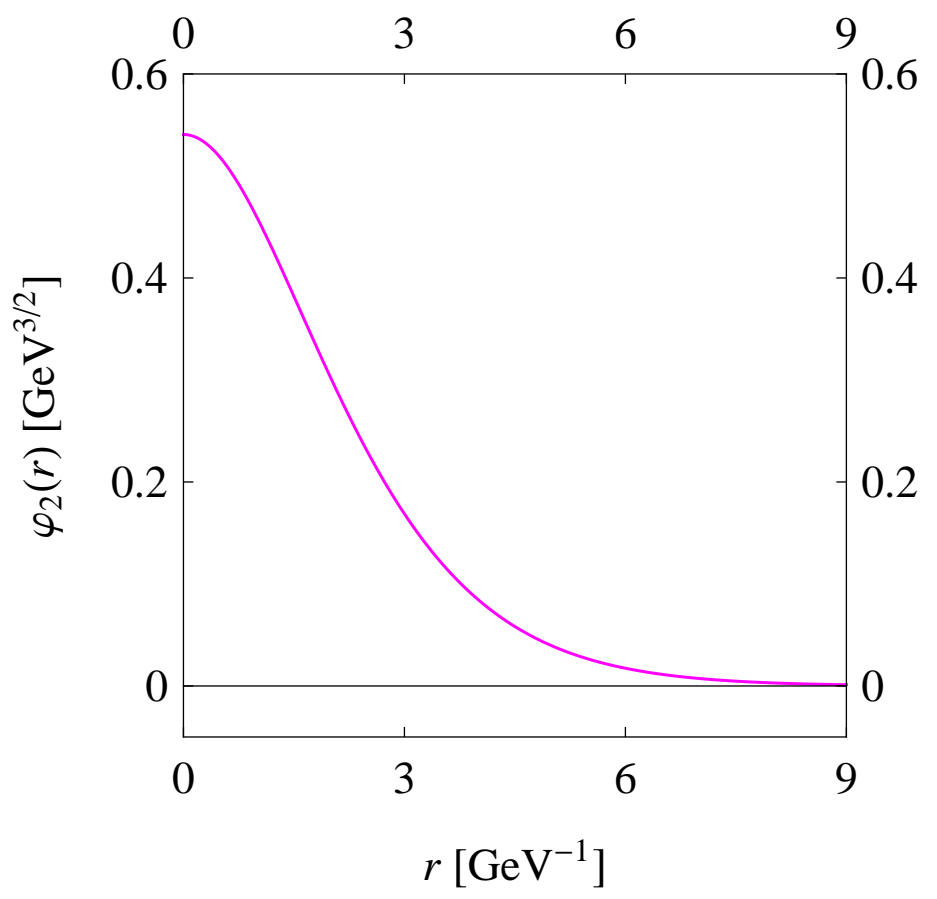

(b)

Figure 2: Nonvanishing radial component function determining the Salpeter amplitude (3.4) of any massless pseudoscalar quark-antiquark bound state governed by our instantaneous Bethe-Salpeter equation (3.1) with Fierz-symmetric Dirac structure (3.2), extracted from the quark propagator functions $M(\underline{k})$ and $Z(\underline{k})$ of Fig. 1 , shown in both (a) momentum-space representation, $\varphi_{2}(p)$, and (b) configuration-space representation, $\varphi_{2}(r)$. 


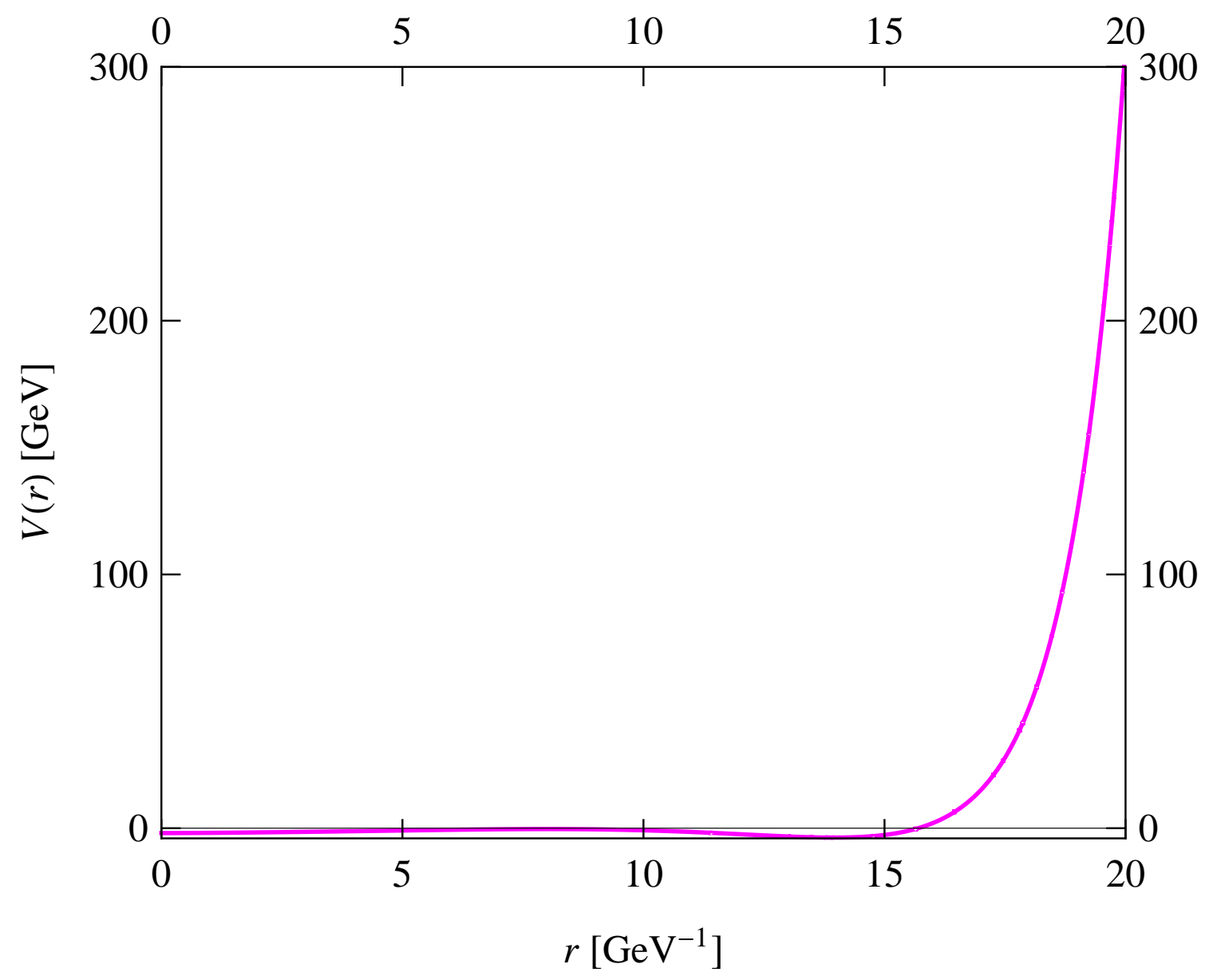

Figure 3: Spherically symmetric effective quark-antiquark interaction potential $V(r)$ that upon insertion into our instantaneous Bethe-Salpeter equation (3.3) trimmed to describe pseudoscalar mesons reproduces, as the ground-state solution to that bound-state problem, the (starting-point) Salpeter component depicted in Fig. 2. Its near flatness close to the origin and steep rise to infinity make $V(r)$ reminiscent of a smoothed square well.

Table 1 presents the very satisfactory outcomes of implementation of the potential of Fig. 3 into our bound-state approach (see, e.g., Refs. [28-30] for corresponding recent Bethe-Salpeter results).

Table 1: Gell-Mann-Oakes-Renner-required features of pseudoscalar quark-antiquark bound states (masses $\widehat{M}_{B}$, decay constants $f_{B}$ and in-meson condensates $\mathbb{C}_{B}$ ) predicted by Eqs. (3.3) for the potential $V(r)$ of Fig. 3, and adequate quark masses $m$ compared with the current-quark values $\bar{m}(\mu)$ in modified minimal subtraction.

\begin{tabular}{rrcccc}
\hline Constituents & $\begin{array}{c}\widehat{M}_{B} \\
{[\mathrm{MeV}]}\end{array}$ & $\begin{array}{c}f_{B} \\
{[\mathrm{MeV}]}\end{array}$ & $\begin{array}{c}\mathbb{C}_{B} \\
{\left[\mathrm{GeV}^{2}\right]}\end{array}$ & $\begin{array}{c}m \\
{[\mathrm{MeV}]}\end{array}$ & $\begin{array}{c}\bar{m}(2 \mathrm{GeV}) \\
{[\mathrm{MeV}][27]}\end{array}$ \\
\hline chiral quarks & 6.8 & 151 & 0.585 & 0.0059 & - \\
u/d quarks & 148.6 & 155 & 0.598 & 2.85 & $3.5_{-0.2}^{+0.5}$ \\
$s$ quarks & 620.7 & 211 & 0.799 & 51.0 & $95_{-3}^{+9}$ \\
\hline
\end{tabular}




\section{References}

[1] W. Lucha and F. F. Schöberl, J. Phys. G 31 (2005) 1133, arXiv:hep-th/0507281.

[2] W. Lucha and F. F. Schöberl, Int. J. Mod. Phys. A 31 (2016) 1650202, arXiv:1606.04781 [hep-ph].

[3] W. Lucha, EPJ Web Conf. 129 (2016) 00047, arXiv:1607.02426 [hep-ph].

[4] W. Lucha, EPJ Web Conf. 137 (2017) 13009, arXiv:1609.01474 [hep-ph].

[5] W. Lucha, preprint HEPHY-PUB 1000/18 (2018), arXiv:1807.06245 [hep-ph].

[6] E. E. Salpeter and H. A. Bethe, Phys. Rev. 84 (1951) 1232.

[7] E. E. Salpeter, Phys. Rev. 87 (1952) 328.

[8] W. Lucha and F. F. Schöberl, Phys. Rev. D 87 (2013) 016009, arXiv:1211.4716 [hep-ph].

[9] W. Lucha, Proc. Sci., EPS-HEP 2013 (2013) 007, arXiv:1308.3130 [hep-ph].

[10] P. Maris, C. D. Roberts, and P. C. Tandy, Phys. Lett. B 420 (1998) 267, arXiv:nucl-th/9707003.

[11] W. Lucha and F. F. Schöberl, Phys. Rev. D 92 (2015) 076005, arXiv:1508.02951 [hep-ph].

[12] W. Lucha and F. F. Schöberl, Phys. Rev. D 93 (2016) 056006, arXiv:1602.02356 [hep-ph].

[13] W. Lucha and F. F. Schöberl, Phys. Rev. D 93 (2016) 096005, arXiv:1603.08745 [hep-ph].

[14] W. Lucha and F. F. Schöberl, Int. J. Mod. Phys. A 33 (2018) 1850047, arXiv:1801.00264 [hep-ph].

[15] P. Maris and P. C. Tandy, Phys. Rev. C 60 (1999) 055214, arXiv:nucl-th/9905056.

[16] P. Maris, in Proceedings of the International Conference on Quark Confinement and the Hadron Spectrum IV, editors W. Lucha and K. Maung Maung (World Scientific, Singapore, 2002), p. 163, arXiv:nucl-th/0009064.

[17] Z.-F. Li, W. Lucha, and F. F. Schöberl, Phys. Rev. D 76 (2007) 125028, arXiv:0707.3202 [hep-ph].

[18] W. Lucha and F. F. Schöberl, Phys. Rev. A 56 (1997) 139, arXiv:hep-ph/9609322.

[19] W. Lucha and F. F. Schöberl, Int. J. Mod. Phys. A 14 (1999) 2309, arXiv:hep-ph/9812368.

[20] W. Lucha, K. Maung Maung, and F. F. Schöberl, Phys. Rev. D 63 (2001) 056002, arXiv:hep-ph/0009185.

[21] W. Lucha, K. Maung Maung, and F. F. Schöberl, Phys. Rev. D 64 (2001) 036007, arXiv:hep-ph/0011235.

[22] W. Lucha and F. F. Schöberl, Recent Res. Dev. Phys. 5 (2004) 1423, arXiv:hep-ph/0408184.

[23] W. Lucha and F. F. Schöberl, Int. J. Mod. Phys. A 29 (2014) 1450057, arXiv:1401.5970 [hep-ph].

[24] W. Lucha and F. F. Schöberl, Int. J. Mod. Phys. A 29 (2014) 1450181, arXiv:1408.4957 [hep-ph].

[25] W. Lucha and F. F. Schöberl, Int. J. Mod. Phys. A 29 (2014) 1450195, arXiv:1410.5241 [hep-ph].

[26] M. Gell-Mann, R. J. Oakes, and B. Renner, Phys. Rev. 175 (1968) 2195.

[27] Particle Data Group (M. Tanabashi et al.), Phys. Rev. D 98 (2018) 030001.

[28] T. Hilger, M. Gómez-Rocha, A. Krassnigg, and W. Lucha, Eur. Phys. J. A 53 (2017) 213, arXiv:1702.06262 [hep-ph].

[29] T. Hilger, M. Gómez-Rocha, A. Krassnigg, and W. Lucha, preprint HEPHY-PUB 1003/18 (2018), arXiv:1807.06245 [hep-ph].

[30] T. Hilger, M. Gómez-Rocha, A. Krassnigg, and W. Lucha, preprint HEPHY-PUB 1008/18 (2018), arXiv:1810.01197 [hep-ph]. 